\title{
Birth route in case of cesarean section in a previous pregnancy
}

\author{
VIA DE PARTO EM CASO DE CESARIANA EM GESTAÇÃO ANTERIOR \\ Authorship: Federação Brasileira das Associações de Ginecologia e Obstetrícia (Febrasgo) and \\ Associação Médica Brasileira (AMB) \\ Participants: Ricardo Simões ${ }^{1,2}$, Wanderley M. Bernardo ${ }^{2}$, Antônio J. Salomão ${ }^{1}$, Edmund C. Baracat ${ }^{1}$ \\ Conflict of interest: no conflict of interest declared
}

${ }^{1}$ Federação Brasileira das Associações de Ginecologia e Obstetrícia (Febrasgo)

${ }^{2}$ Programa Diretrizes da AMB

The Guidelines Project, an initiative of the Brazilian Medical Association, aims to combine information from the medical field in order to standardize procedures to assist the reasoning and decision-making of doctors.

The information provided through this project must be assessed and criticized by the physician responsible for the conduct that will be adopted, depending on the conditions and the clinical status of each patient.

\section{INTRODUCTION}

Over the past decades, the increase in cesarean section rates worldwide, especially in developed countries, has been evident. Nearly a third of all births in the US occur by cesarean section and the proportion of both, first cesarean and repeat cesarean, is still increasing every year $(\mathbf{A})^{1}(\mathbf{D}) .^{3}$

Cesarean delivery is a relatively simple procedure, but with inherent risks to its indication that predispose to various complications such as puerperal infection, ectopic pregnancy and thromboembolic events, especially when repeated. The risk of maternal death is increased, as much as the occurrence of infection, hemorrhage and anesthetic complications, which also increases maternal morbidity compared to vaginal delivery. Late complications, especially poor placental implantation in the subsequent pregnancy (placenta with low insertion and varying degrees of placenta accreta), are directly related to a history of cesarean section $(\mathbf{B}){ }^{2}$ Maternal morbidity increases in response to each additional cesarean section, especially for women with three or more cesarean sections who have a high risk of low insertion placenta, placenta accreta and hysterectomy $(\mathbf{A}){ }^{1}$

With regard to the fetus, although cesarean section can save lives if indicated due to cord prolapse, placenta previa, cephalopelvic disproportion and fetal distress, for example, it can also lead to increased risk of iatrogenic prematurity and neonatal respiratory distress when performed without a precise indication $(\mathbf{B}){ }^{2}$

Nevertheless, even though the complications related to trial of labor (TOL) in pregnant women with previous cesarean section are minimal, they are not ruled out and must, thus, be taken into consideration.

\section{Овjective}

The objective of this review is to provide the best evidence available today on maternal morbidity and mortality re- garding birth route (vaginal delivery or cesarean section) in case of cesarean section in a previous pregnancy.

\section{Material ANd methods}

Evidence used to analyze maternal morbidity and mortality regarding birth route chosen in case of cesarean section in a previous pregnancy was obtained as follows: preparation of a clinical question, structuring of the question, search of evidence, critical appraisal and evidence selection.

\section{Clinical question}

Is performance of elective cesarean section in case of cesarean delivery in a previous pregnancy related with less maternal morbidity and mortality compared with vaginal delivery?

\section{Structured question}

The clinical question is structured according to the P.I.C.O. components (P [Patient]; I [Intervention]; C [Comparison]; O [Outcome]).

- P: C-section in previous pregnancy

- I: C-section

- C: Vaginal delivery

- O: Maternal morbidity and mortality

Scientific databases consulted

The scientific databases consulted were: PubMed-Medline and Cochrane. Manual search based on reviewed references (narrative or systematic) was also performed.

Strategies for search of evidence

PubMed-Medline

Strategy: (cesarean section, repeat OR repeat cesarean section OR cesarean sections, repeat OR repeat cesarean 
sections OR section, repeat cesarean OR sections, repeat cesarean) AND (trial of labor OR vaginal birth after cesarean $O R$ vaginal birth after cesareans $O R$ vaginal births after cesarean).

\section{Cochrane}

Strategy: repeat cesarean section.

Studies retrieved (5/1/2014) (Table 1)

TABLE 1 Number of studies retrieved according to the search strategies used for each scientific database.

\begin{tabular}{ll} 
Database & Number of studies \\
\hline Primary & \\
\hline PubMed-Medline & 584 \\
\hline Cochrane & 43
\end{tabular}

\section{Inclusion criteria for studies retrieved}

Selection of studies, assessment of titles and abstracts obtained from the search strategy in the consulted databases was conducted by two researchers with skills in the preparation of systematic reviews, both independent and blinded, strictly observing the inclusion and exclusion criteria previously established (see item 2 ). All potentially relevant studies were identified. Whenever the title and the summary were not enlightening, researchers sought the full article.

\section{Study design}

Narrative reviews, case reports, case series and studies presenting preliminary results were excluded from the assessment. Systematic reviews and meta-analyzes were used with the basic purpose of recovering references that perhaps had been missed in the initial search strategy. Studies designed as cohort or controlled clinical trials (randomized or not) were included.

Cohort study was defined as those with follow-up of patients, equal history, and analysis of prognostic outcomes.

Controlled clinical trials were evaluated according to the Jadad score. ${ }^{4}$

\section{P.I.C.O. components}

- Patient: women in labor of a term singleton fetus in cephalic presentation, who had babies delivered by cesarean section in previous births (cesarean delivery performed by means of transverse uterine section).

- Intervention: elective cesarean section.

- Comparison: vaginal delivery.

- Outcomes related to maternal morbidity and mortality: maternal death, uterine rupture, hysterectomy scar dehiscence (total or partial), hemorrhagic complications (bleeding in the intra- and post-partum period requiring blood transfusion), retention of placental remains, hysterectomy related to any birth complications, vulvar or perineal hematoma (requiring surgery), thromboembolic events, surgical wound complications (wound infection, dehiscence or pain), puerperal infection, bowel, bladder or ureter injury requiring surgical treatment, occurrence of fistula involving the urinary, genital or gastrointestinal tracts, pulmonary edema, postpartum depression. Late maternal outcomes include complications in breastfeeding, perineal pain, abdominal pain, dyspareunia, urinary incontinence, fecal incontinence, genital dystopia, occurrence of low insertion of placenta or placenta accreta/percreta in future pregnancies.

\section{Language}

We included studies available in Portuguese, English, French or Spanish.

\section{According to publication}

Only studies with full text available were considered for critical assessment.

\section{Studies selected in the first assessment}

After entering the search strategy in the primary databases (PubMed-Medline and Cochrane), the assessment of titles and abstracts led to the selection of nine studies.

\section{Evidence selected after critical evaluation and exhibition} of results

The studies considered for full text reading were critically assessed according to inclusion and exclusion criteria, study design, P.I.C.O., language and availability of the full text (items 1, 2, 3 and 4).

Results pertaining clinical status will be displayed individually showing the following items: clinical question, number of studies selected (according to inclusion criteria), description of the studies (Table 2), results and summary of the available evidence.

References related to the studies included are shown in Table 4.

After applying the inclusion and exclusion criteria, the evidence selected in the search and defined as randomized controlled trials (RCT) were subjected to an appropriate checklist for critical assessment (Table 3). Critical assessment of RCTs allows to classify them according to the Jadad score, so that trials with Jadad $<3$ are considered inconsistent $(\mathbf{B})$, and those with scores $\geq 3$, consis- 
tent (A). For critical analysis of non-randomized studies, including prospective observational studies, we used the Newcastle-Ottawa scale. ${ }^{5}$

For results with evidence available, whenever possible the following specific items are defined: population, intervention, outcomes, the presence or absence of benefit and/or damage and controversies.

Cost issues will not be included in the results.

The results will be presented preferably in absolute data, absolute risk, number needed to treat (NNT) or number needed to harm (NNH), and occasionally in mean and standard deviation.

References related to included and excluded studies are shown in section References.

\begin{tabular}{l} 
TABLE 2 Worksheet used for description of studies \\
included and exposure of the results. \\
Worksheet for description of studies and exposure of the \\
results \\
\hline Evidence included \\
\hline Study design \\
\hline Population selected \\
\hline Time of follow-up \\
\hline Outcomes considered \\
\hline Expression of results: percentage, risk, odds, hazard ratio
\end{tabular}

TABLE 3 Critical assessment script for randomized controlled trials (checklist).

\begin{tabular}{ll} 
Study data & Sample size calculation \\
Reference, study design, Jadad, & Estimated differences, power, \\
strength of evidence & $\begin{array}{l}\text { significance level, total number } \\
\text { of patients }\end{array}$ \\
\hline Patient selection & Patients \\
Inclusion and exclusion criteria & $\begin{array}{l}\text { Recruited, randomized, } \\
\text { prognostic differences }\end{array}$ \\
\hline Randomization & Patient follow-up \\
Description and blinded allocation & Time, losses, migration \\
\hline Treatment protocol & Analysis \\
Intervention, control and blinding & $\begin{array}{l}\text { Intention to treat, analyzes of } \\
\text { intervention and control }\end{array}$ \\
\hline Outcomes considered & Result \\
Primary, secondary, measuring & Benefit or harm in absolute \\
instrument of the outcome of & data, benefit or harm on \\
interest & average \\
\hline
\end{tabular}

\section{Clinical question}

Is performance of elective cesarean section in case of cesarean delivery in a previous pregnancy related with less maternal morbidity and mortality compared with vaginal deliver?

Evidence selected

TABLE 4 Selection process.

\begin{tabular}{lc} 
Type of publication & Included \\
\hline Randomized clinical trials & \\
Current cohort studies & $9^{6-14}$ \\
Non-current cohort studies & \\
\hline
\end{tabular}

The main reasons for the exclusion of studies were: study design other than observational longitudinal clinical trials (retrospective or prospective) or experimental (controlled clinical trials, randomized or not); absence of the full text; selection for the performance of elective cesarean section in women not considered eligible to trial of labor; studies that did not assess the two approaches simultaneously; studies that included populations with unique characteristics such as twin pregnancies, breech delivery and two or more cesarean section scars.

Results of the evidence selected

Of the 584 articles initially retrieved, nine were selected to support the synthesis of evidence regarding maternal morbidity and mortality related to type of delivery in case of cesarean section in a previous pregnancy. Studies included are shown in Table 4.

1. Crowther CA, et al. (B). ${ }^{6}$

- Design: concurrent observational longitudinal study with nested randomized clinical trial.

- Population: 2,345 pregnant women with one previous cesarean section and eligible for planned vaginal delivery were recruited from 14 centers. Patients with fetuses at term (gestational age $\geq 37$ weeks) were allocated according to preference $(n=2,323)$ or randomized $(n=22)$ into groups for planned vaginal delivery or cesarean section.

- Outcome: to assess, as the primary outcome, perinatal and neonatal morbidity and mortality, excluding cases related to fatal congenital anomalies. The analysis included birth trauma (bone fractures, subdural hematoma, brain or intraventricular hemorrhage), spinal cord injury, peripheral nerve injury, seizures (within 24 hours of birth or need for two or more drugs to control) Apgar score $<4$ at five minutes, need for assisted ventilation, stay in neonatal intensive care unit for longer than four days. 
As a secondary outcome, maternal morbidity and mortality were assessed, including maternal death, uterine rupture, severe bleeding, need for blood transfusion, need for uterine curettage due to presence of ovular debris, hysterectomy, need for surgical treatment of vulvar or perineal hematoma, pulmonary embolism, thromboembolic events, pneumonia, surgical wound infection, dehiscence, bowel, bladder or ureter injury requiring surgical treatment, genital tract fistulae.

- Results: of the 2,345 pregnant women, 1,108 underwent cesarean elective delivery (including 10 randomized to such procedure), while 1,237 underwent planned vaginal delivery (with 12 cases being randomized to this type of delivery). In this study, the authors found that among women undergoing cesarean delivery, outcomes related to perinatal and neonatal morbidity and mortality were significantly less frequent compared to women undergoing planned vaginal delivery ( 0.9 versus $2.4 \%$, respectively). With respect to secondary outcomes relating to maternal morbidity and mortality, there was no significant difference between groups ( $R R=0.69$ with $95 \% \mathrm{CI}$ : 0.46 to 1.05$)$. It is worth noting that the data reported here refer to a cohort study and, given the small sample of patients randomized, risks cannot be estimated.

2. Landon MB, et al. (B). ${ }^{7}$

- Design: multicenter, prospective, observational longitudinal study (1999 to 2002).

- Population: out of 45,988 women presenting singleton pregnancies and history of previous cesarean delivery, $\mathrm{n}=17,898$ underwent trial of labor and 15,801 underwent elective cesarean section. We must underscore that heterogeneity between these two groups existed mainly regarding maternal age, ethnicity, smoking status, body mass index, history of vaginal delivery, number of previous cesarean sections, the presence of maternal or obstetric disease, weight and gestational age at birth.

- Outcome: to assess maternal, perinatal and neonatal morbidity and mortality.

- Results: over the four years, higher maternal mortality was observed, with regard to the need for blood transfusion and occurrence of endometriosis among women undergoing trial of labor to the detriment of elective cesarean section $(\mathrm{RR}=0.589$ with $95 \%$ CI: 0.484 to 0.716 , and $R R=0.624$ with $95 \%$ CI: 0.540 to 0.722 , respectively). Analyzing cas- es of uterine rupture, the authors found that 124 cases were reported among women undergoing trial of labor versus the absence of this outcome for births that occurred by means of cesarean section. Regarding the occurrence of maternal death and need for hysterectomy, there was no difference between groups.

In neonatal morbidity and mortality analysis, the authors observed that hypoxic-ischemic encephalopathy occurrences were among the babies born vaginally (12 cases) versus the absence of this outcome for elective cesarean section.

3. Eriksen NL, et al. (B). ${ }^{8}$

- Design: non-concurrent observational longitudinal study.

- Population: records of 152 pregnant women with a previous cesarean section were reviewed. 68 women underwent elective cesarean section, while 73 underwent planned vaginal delivery.

- Outcome: maternal, perinatal and neonatal morbidity and mortality.

- Results: regarding maternal and neonatal morbidity and mortality, there were no differences between the two types of delivery.

4. Flamm BL, et al. (B). ${ }^{9}$

- Design: prospective observational longitudinal study.

- Population: of the 7,229 women with a previous cesarean section, 5,022 underwent trial of labor, and 2,207 underwent elective cesarean section.

- Outcome: maternal morbidity and mortality.

- Results: the need for blood transfusion and the incidence of febrile complications during the postpartum period were significantly higher among women undergoing elective cesarean section.

5. Loebel G, et al. (B). ${ }^{10}$

- Design: non-concurrent observational longitudinal study.

- Population: records of 1,408 pregnant women (pregnant women with singleton pregnancy at term and a previous cesarean section) were reviewed.

- Outcome: maternal, perinatal and neonatal morbidity and mortality.

- Results: regarding maternal morbidity and mortality, there was no significant difference in the need for blood transfusion, infection, uterine rupture and surgical lesion between the two birth 
routes. On the other hand, analyzing neonatal morbidity and mortality, the authors were able to identify more frequent respiratory complications among those born by cesarean section $(\mathrm{p}<0.05)$.

6. Blanchette $\mathrm{H}$, et al $(\mathbf{B}){ }^{11}$

- Design: concurrent observational longitudinal study.

- Population: prospective study over 4 years including all births to women who underwent previous cesarean delivery $(\mathrm{n}=1.481)$. Comparison was made between those who chose to repeat cesarean section $(n=727)$ and the women who opted for TOL $(n=754)$.

- Outcome: maternal morbidity and mortality.

- Results: regarding the occurrence of uterine rupture, need for blood transfusion, and hysterectomy, the authors found a higher frequency among women undergoing trial of labor. However, among these women, the maternal mortality rate was lower compared to those undergoing elective cesarean section.

7. Paterson CM, et al. (B). ${ }^{12}$

- Design: non-concurrent observational longitudinal study.

- Population: records of 1,059 women in singleton labor and with a previous cesarean section.

- Outcome: postnatal morbidity and mortality.

- Results: 395 women underwent elective cesarean section and 664 underwent trial of labor. $71 \%$ of the women undergoing trial of labor gave birth by vaginal delivery. In this study, higher maternal morbidity (postnatal infection) was identified both in patients undergoing elective cesarean delivery and in those with cesarean section performed due to failure to progress, compared to vaginal delivery.

8. Wen SW, et al. (B). ${ }^{13}$

- Design: non-concurrent observational longitudinal study (1988 to 2000).

- Population: records of 308,755 women with a history of previous cesarean section were reviewed.

- Outcome: maternal morbidity and mortality.

- Results: the authors found that the risk of uterine rupture was higher among women undergoing trial of labor compared to those undergoing elective cesarean section ( $\mathrm{RR}=2.59$ with $95 \% \mathrm{CI}$ : 2.31 to 2.91). Regarding the need for hysterectomy, there was no significant difference between groups.

Analyzing the occurrence of postpartum infection and need for blood transfusion, the authors found a lower risk of these outcomes among women undergoing trial of labor ( $\mathrm{RR}=0.81$ with $95 \% \mathrm{CI}$ : 0.724 to 0.908 , and $\mathrm{RR}=1.27$ with $95 \% \mathrm{CI}$ : 1.068 to 1.521 ).

9. McMahon MJ, et al. (B). ${ }^{14}$

- Design: non-concurrent observational longitudinal study (1986 to 1992).

- Population: records of 6,138 women with a history of previous cesarean section were reviewed (undergoing trial of labor $[n=3,249]$ or elective cesarean section $[\mathrm{n}=2,889])$.

- Outcome: maternal and neonatal morbidity and mortality.

- Results: the authors found that the risk of severe complications including uterine rupture, hysterectomy and need for surgical lesion was greater among patients undergoing trial of labor compared to those undergoing elective cesarean section ( $\mathrm{RR}=1.96$ with $95 \% \mathrm{CI}$ : 1.18 to 3.26 ). Regarding minor complications (need for blood transfusion, puerperal fever and surgical wound infection), there was no significant difference between groups ( $\mathrm{RR}=0.82$ with $95 \% \mathrm{CI}$ : 0.68 to 1.0 ). Based on the analysis of Apgar scores, need for admission to neonatal intensive care unit and perinatal mortality, there was no difference between groups.

\section{Discussion}

Based on the above, we have identified a lack of controlled studies analyzing the best type of delivery in cases of cesarean section in a previous pregnancy. Supported mainly by observational studies, most of them retrospective, current evidence analyzing the outcomes of maternal morbidity and mortality related to the type of delivery in this clinical context is fragile, especially on account of biases inherent to this particular type of study $(\mathbf{B}){ }^{6-14}$ Facts that contribute to the difficulty in finding definitive evidence about the best type of delivery include: heterogeneity in terms of methodology employed to measure the outcomes; distinct characteristics of the women enrolled (body mass index, age, race, obstetric history, gestational age) and their babies (especially with respect to birth weight); and different study durations, from start to end of the intervention, plus follow-up period (in which the conduction of labor in terms of technologies and rates of trial of labor differed). This creates difficulties to analyze the true magnitude of the benefits and risks in cases of cesarean section in a previous pregnancy, without clarifying the doubts about the real superiority of elective cesarean section indication in these 
patients compared to trial of labor in terms of effectiveness and safety.

The study by Landon et al. illustrates the difficulties presented above because, despite being the most important prospective observational longitudinal study conducted to evaluate this outcome, it has biases inherent to the study design, which are easily identified and compromise the analysis (B). ${ }^{7}$ In this study, despite the fact that the women included in both groups were mostly obese, with $\mathrm{BMI}>30 \mathrm{Kg} / \mathrm{m}^{2}$, there was heterogeneity especially regarding maternal age, ethnicity, smoking status, obstetric history, factors leading to indication of the first cesarean delivery, obstetric diseases, gestational age, and fetal weight at birth. The study was multicentric, and thus trial of labor rates varied greatly among different centers included (ranging from 18 to 63\%), with a decrease throughout the study period (in 1999, there were on average $48 \%$ of patients undergoing trial of labor, falling to $30 \%$, in 2002). Despite that, we have identified a significant increase in the number of maternal complications in cases of pregnant women undergoing trial of labor (such as need for blood transfusion, diagnosis of endometriosis, and uterine rupture), compared to patients referred for elective cesarean section, especially in cases where there was failure to progress. However, due to the heterogeneity of the groups and the exclusion of patients who underwent elective cesarean section at the beginning of labor, the interpretation of these results has limited range $(\mathbf{B}){ }^{?}$

In 2012, another concurrent observational longitudinal study, aimed at analyzing the outcomes related to type of delivery on maternal morbidity and mortality (here considered as a secondary outcome), was published ${ }^{6}(\mathbf{B})$. Also multicentric, this study was less questioned in terms of methodology compared to the first trial shown above, since the groups were homogeneous regarding intention to treat (excluding body mass index, socioeconomic status and obstetric indication of first cesarean section). There was no significant difference between the groups; however, it should be noted that the outcome of interest (maternal morbidity and mortality expressed by the occurrence of at least one serious complication such as death, uterine rupture, postoperative complications, bleeding, thromboembolism and infection) was characterized as secondary $(\mathbf{B}){ }^{6}$

Also in 2012, a study (secondary analysis of the cohort study by Landon et al., published in 2004) that adopted a multiple logistic regression model was published. Using a propensity score with control of presented confounding factors (heterogeneity between groups), the study had the main objective of comparing the progno- sis by type of delivery in cases with previous cesarean section. Even after this analysis, consistent with findings reported in the initial study, it was found that elective cesarean indication was associated, to the detriment of trial of labor, to a significant reduction of maternal complications $(\mathbf{B}){ }^{15}$

\section{Final ReCommendations}

Based on the evidence available at present, trial of labor (TOL) is a reasonable option for women with a previous cesarean section (performed through transverse uterine section).

The available data derived from observational longitudinal studies (concurrent or not) show that both labor and elective cesarean section in pregnant women (with a single fetus in cephalic presentation) with one previous cesarean section are associated with significant risks and benefits, which differ for the mother and the fetus (risk of uterine rupture, febrile morbidity, need for blood transfusion and hysterectomy).

Characteristics that are clearly associated with a favorable progress of trial of labor are: indication of the first cesarean section (non-recurring reason) and a history of vaginal delivery, in the case of multiparous women with a previous cesarean section.

Since data is not conclusive as to the best type of delivery in this context, recognition of the prognostic factors associated with the failure of the trial of labor should be helpful, as an attempt to select more properly women who should effectively undergo such procedure.

\section{References}

1. Marshall NE, Fu R, Guise J-M. Impact of multiple cesarean deliveries on maternal morbidity: a systematic review. Am J Obstet Gynecol. 2011; 205:262.e1-8.

2. Matias JP, Parpinelli MA, Cecatti JG. A prova de trabalho e a via de parto em primíparas com uma cesárea anterior. Rev Assoc Med Bras. 2007; 53(2): 109-15.

3. NIH Consensus Development Conference Statement on Vaginal Birth After Cesarean: new insights 2010;27(3)

4. Jadad AR, Moore RA, Carroll D, Jenkinson C, Reynolds DJ, Gavaghan DJ, et al. Assessing the quality of reports of randomized clinical trials: is blinding necessary? Control Clin Trials. 1996; 17(1):1-12.

5. Wells GA, Shea B, O'Connell D, Peterson J, Welch V, Losos M, et al. The Newcastle-Ottawa Scale (NOS) for assessing the quality of nonrandomised studies in meta-analyses. Available at: www.ohri.ca/programs/clinical_ epidemiology/oxford.asp. Acessado: 10 fev 2014

6. Crowther CA, Dodd JM, Hiller JE, Haslam RR, Robinson JS; Birth After Caesarean Study Group. Planned vaginal birth or elective repeat caesarean: patient preference restricted cohort with nested randomised trial. PLoS Med. 2012; 9(3):e1001192.

7. Landon MB, Hauth JC, Leveno KJ, Spong CY, Leindecker S, Varner MW, et al.; National Institute of Child Health and Human Development MaternalFetal Medicine Units Network. Maternal and perinatal outcomes associated with a trial of labor after prior cesarean delivery. N Engl J Med. 2004; 351(25):2581-9. 
8. Eriksen NL, Buttino L Jr. Vaginal birth after cesarean: a comparison of maternal and neonatal morbidity to elective repeat cesarean section. Am J Perinatol. 1989; 6(4):375-9.

9. Flamm BL, Goings JR, Liu Y, Wolde-Tsadik G. Elective repeat cesarean delivery versus trial of labor: a prospective multicenter study. Obstet Gynecol. 1994; 83(6):927-32

10. Loebel G, Zelop CM, Egan JF, Wax J. Maternal and neonatal morbidity after elective repeat Cesarean delivery versus a trial of labor after previous Cesarean delivery in a community teaching hospital. J Matern Fetal Neonatal Med. 2004; 15(4):243-6.

11. Blanchette $\mathrm{H}$, Blanchette $\mathrm{M}, \mathrm{McCabe} J$, Vincent $\mathrm{S}$. Is vaginal birth after cesarean safe? Experience at a community hospital. Am J Obstet Gynecol. 2001; 184(7):1478-84; discussion 1484-7.

12. Paterson CM, Saunders NJ. Mode of delivery after one caesarean section: audit of current practice in a health region. BMJ. 1991; 303(6806):818-21.
13. Wen SW, Rusen ID, Walker M, Liston R, Kramer MS, Baskett T, Heaman M, Liu S; Maternal Health Study Group, Canadian Perinatal Surveillance System. Comparison of maternal mortality and morbidity between trial of labor and elective cesarean section among women with previous cesarean delivery. Am J Obstet Gynecol. 2004; 191(4):1263-9.

14. McMahon MJ, Luther ER, Bowes WA Jr, Olshan AF. Comparison of a trial of labor with an elective second cesarean section. N Engl J Med. 1996; 335(10):689-95.

15. Gilbert SA, Grobman WA, Landon MB, Spong CY, Rouse DJ, Leveno KJ, et al.; Eunice Kennedy Shriver National Institute of Child Health and Human Development Maternal-Fetal Medicine Units Network. Elective repeat cesarean delivery compared with spontaneous trial of labor after a prior cesarean delivery: a propensity score analysis. Am J Obstet Gynecol. 2012; 206(4):311.e1-9. 